\title{
Heterodyne instrumentation upgrade at the Caltech Submillimeter Observatory
}

Jacob W. Kooi, Attila Kovacs, Steven M. Kaye, J. Dama, Michael L. Edgar, et al.

Jacob W. Kooi, Attila Kovacs, Steven M. Kaye, J. Dama, Michael L. Edgar, Jonas Zmuidzinas, Thomas G. Phillips, "Heterodyne instrumentation upgrade at the Caltech Submillimeter Observatory," Proc. SPIE 4855, Millimeter and Submillimeter Detectors for Astronomy, (17 February 2003); doi: $10.1117 / 12.459114$

Event: Astronomical Telescopes and Instrumentation, 2002, Waikoloa, Hawai'i, United States 


\title{
Heterodyne Instrumentation Upgrade at the Caltech Submmillimeter Observatory
}

\author{
J. W. Kooi, A. Kovács, S. Kaye, J. Dama, M. L. Edgar, J. Zmuidzinas, and T.G. Phillips \\ California Institute of Technology, MS 320-47, Pasadena, Ca 91125, USA
}

\begin{abstract}
Under development at the Caltech Submillimeter Observatory is a dual polarization, continuous comparison (correlation) receiver. The instrument has two beams on the sky; a reference and a signal beam. Using only cooled reflecting optics, two polarizing grids, and a quadrature hybrid coupler, the sky beams are coupled to four tunerless SIS mixers (both polarizations). The 4-8 GHz mixer IF outputs are, after amplification, correlated against each other. In principle, this technique results in flat baselines with very low RMS noise and is especially well suited for high redshift Galaxy work. At the same time an upgrade is planned to the existing facility heterodyne instrumentation. Dual frequency mode receivers are under development for the 230/460 $\mathrm{GHz}$ and 345/660 $\mathrm{GHz}$ atmospheric windows. The higher frequency receivers are implemented in a balanced configuration, which reduces both the LO power requirement and noise. Each mixer has $4 \mathrm{GHz}$ of IF bandwidth and can be controled remotely.
\end{abstract}

Not only do these changes greatly enhance the spectroscopic capabilities of the CSO, they also enable the observatory to be integrated into the Harvard-Smithsonian Submillimeter Array (SMA) as an additional baseline.

Keywords: Radial probe, full-height waveguide to thin-film microstrip transition, split-block, superconductinginsulating-superconducting (SIS) tunnel junction, broad bandwidth quadrature waveguide hybrid, DC-break, IF match.

\section{INTRODUCTION}

The existing SIS waveguide receivers at the CSO, except for the 800-950 GHz Quasi-Optical receiver, all use waveguide tuners to achieve sensitivities a few times the quantum noise limit. The existing IF bandwidth for these instruments is $1 \mathrm{GHz}$. Every one of these receivers has pioneered the submillimeter field, however in spite of the state-of-the-art nature of the receivers; the astronomy is demanding more capability in terms of sensitivity, bandwidth, stability, and ease of use. To fill this need, we are planning a significant upgrade to these instruments. Four tunerless waveguide receivers have been designed to cover the entire $180-720 \mathrm{GHz}$ frequency range. The IF bandwidth of the CSO receivers will increase from $1 \mathrm{GHz}$ to $4 \mathrm{GHz}$, and interfaces to a newly acquired $4 \times 1 \mathrm{GHz}$ wide bandwidth, University of Köln build, Acousto-Optical Spectrometer (AOS). ${ }^{1-3}$ Dual frequency observations $(230 / 460 \mathrm{GHz}$ and $345 / 660 \mathrm{GHz}$ atmospheric frequency bands) can be configured by routing the respective receiver IF output channels to the Köln built hybrid AOS. Because no tuners are needed, the orientation and placement of the mixer blocks has become arbitrary. This, and the new bias electronics, makes it realistic to implement dual frequency (2 colors) observations. Not only provides this scheme a new mode of observation for the CSO, it will also be a great help in pointing the telescope in mediocre weather.

In addition to the new receivers, we are constructing a specialized 4-channel, dual polarization, continuous comparison (correlation) receiver to operate in the $280-420 \mathrm{GHz}$ atmospheric window. As for the backend correlator, we anticipate to use WASP, ${ }^{4,5}$ a $16 \mathrm{MHz} /$ channel analog spectrometer from the University of Maryland. The heterodyne facility instrumentation and the specialized correlation receiver have much of their hardware in common. Design of the mixer blocks, associated waveguide hybrid couplers, and the IF amplifier chain is nearly complete. Delivery of all the components is expected in the spring of 2003. Remote control

Further author information: (Send correspondence to Jacob W. Kooi)

Jacob W. Kooi E-mail: kooi@submm.caltech.edu, Telephone: 16263954286

Millimeter and Submillimeter Detectors for Astronomy, Thomas G. Phillips, Jonas Zmuidzinas, 


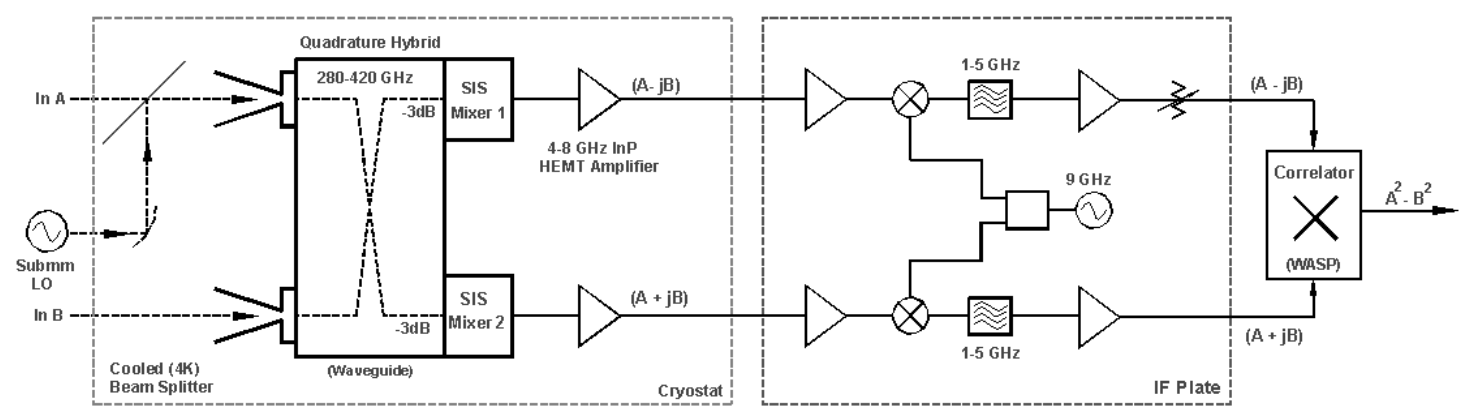

Figure 1. Block Diagram of the Continuous Comparison (Correlation) Receiver. Only one of the the polarizations is shown.

receiver electronics and software is expected to reach an operational level in the summer of 2003. The Cryostat that houses the first of the upgrades, namely a dual frequency $345 / 650 \mathrm{GHz}$ instrument, is currently being worked on at the CSO laboratory in Hilo, Hawaii, and is expected to be ready by the end of 2002 .

The SIS tunnel junctions needed for the upgrade have been developed by Kovács et. al.. ${ }^{6}$ These devices will be submitted to JPL for fabrication in the fall of 2002. Installation of the new instrumentation, pending timely delivery of all the new hardware, is anticipated to commence in the summer of 2003.

\section{A 280-420 GHZ DUAL POLARIZATION, CONTINUOUS COMPARISON (CORRELATION) RECEIVER}

Probably the most popular receiver at the CSO is the facility $280-420 \mathrm{GHz}$ system. However, for many distant galaxy projects, an improvement in sensitivity of a factor of 5-6 is needed. This can only be obtained by use of a more sophisticated design. This is best achieved by constructing a 4-detector receiver with dual polarization and on-off source detectors operating in a correlation or continuous comparison mode (Predmore et al. $1985^{7}$ ). This design rejects signals present in both channels. The block diagram for one polarization of this design is shown in Fig. 1.

It is expected that the rejection of gain variation, plus extra signal from continuous comparison and dual polarization, will indeed provide the needed performance gain in sensitivity of approximately 6 over the existing waveguide tuned $280-420 \mathrm{GHz}$ receiver. Further advantages will accrue from the $4 \mathrm{GHz}$ bandwidth compared to the present $1 \mathrm{GHz}$. The $280-420 \mathrm{GHz}$ band is chosen for this first case, not only because of the astronomical opportunities, but also because there is no shortage of local oscillator power for four junctions, waveguide techniques work well here, atmospheric and optical loss is relatively low, telescope efficiency is high (74\%), and it is judged most likely to provide search opportunities for redshifted CII lines $(z=3.7-5.8)$.

It should be noted that yet more sensitivity can be obtained for point source detection, with a single dish system, by (in addition to the above) also separating the sidebands. To do all this, for a single pixel on the sky would require 8 detectors and either 4 or 8 wideband backends, depending on the technique used. Since there is good atmoshperic transparency over much of the $280-420 \mathrm{GHz}$ frequency band at the CSO, dual polarization and instantaneous on-off detections are probably better choices than sideband separation. In this case, 2 rather than 4 backends can be used with the correlation receiver. Moreover, it has been shown (Lamb et al. ${ }^{8}$ ) that the actual advantage gained by separating the sidebands is very quickly diminished in any real, non-zero temperature system.

For these reasons, the decision has been made to use only four detectors and two wideband spectrometers, configured in a correlating mode. This is very important for rejecting gain variation, which is the primary problem facing wideband detection with SIS receivers. The gain in sensitivity is $\sqrt{2}$ for both polarizations, at least $\sqrt{2}$ for both on and off detection (depending on how much this suppresses gain variation effects). Given 
an optical depth $\left(\tau_{a t m}\right)$ of 0.1 at $345 \mathrm{GHz}$, a $74 \%$ main beam efficiency of the telescope, a DSB receiver noise temperature of $50 \mathrm{~K}$, and $100 \mathrm{MHz}$ of spectral resolution, we estimate an RMS noise level in the order of $85 \mu \mathrm{K}$ in 8 hours of integration time. The latter assumes that the noise integrates down perfectly with root time, which is not the case for the existing receivers.

$T_{\text {sys }}(S S B)$ for a single pixel can be estimated as:

$$
T_{\text {sys }}(S S B)=2 * \frac{\left(T_{r e c}(D S B)+T_{a t m} *\left(1-e^{-\tau_{a t m}}\right)+T_{\text {spill }}\right)}{\eta_{m b} * \eta_{\text {spill }} * e^{-A * \tau_{a t m}}}
$$

Where $\eta_{m b}$ represents the main beam efficiency $(0.74), \eta_{\text {spill }}=0.95, T_{\text {spill }}=14 \mathrm{~K}$, and A the airmass. Since

$$
T_{a t m} \approx 0.95 * T_{a m b}
$$

and $T_{a m b}$ is typically $270 \mathrm{~K}$, we find from the radiometer equation, ${ }^{9}$ that the rms noise of the correlation receiver equals $\approx 85 \mu \mathrm{K}$ in 8 hours of integration time.

$$
\sigma=\frac{T_{\text {sys }}(S S B)}{\sqrt{\left(n * B * \tau_{\text {int }}\right)}}
$$

Here $\mathrm{n}=4$, and is the expected improvement of the dual polarization correlation receiver over a single pixel receiver.

\section{UPGRADES TO THE EXISTING FACILITY HETERODYNE INSTRUMENTATION}

Having developed all the mixer hardware of the proposed tunerless $280-420 \mathrm{GHz}$ correlation receiver, it is only natural to extend this effort to the existing heterodyne instrumentation. As discussed, the current CSO facility heterodyne receivers consist, with the exception of the all NbTiN based 780-950 GHz quasi-optical receiver, of tuned waveguide mixers with a $1 \mathrm{GHz}$ IF bandwidth. The instrumentation upgrade constitutes the development of ten tunerless, full height waveguide, mixers and associated IF/RF hardware. The frequency bands of the new sidecab receivers are as follows: $180-280 \mathrm{GHz}, 280-420 \mathrm{GHz}, 380-520 \mathrm{GHz}, 580-720 \mathrm{GHz}$. Also planned is an upgrade of the NbTiN quasi-optical twin-slot receiver IF passband to $4 \mathrm{GHz}$.

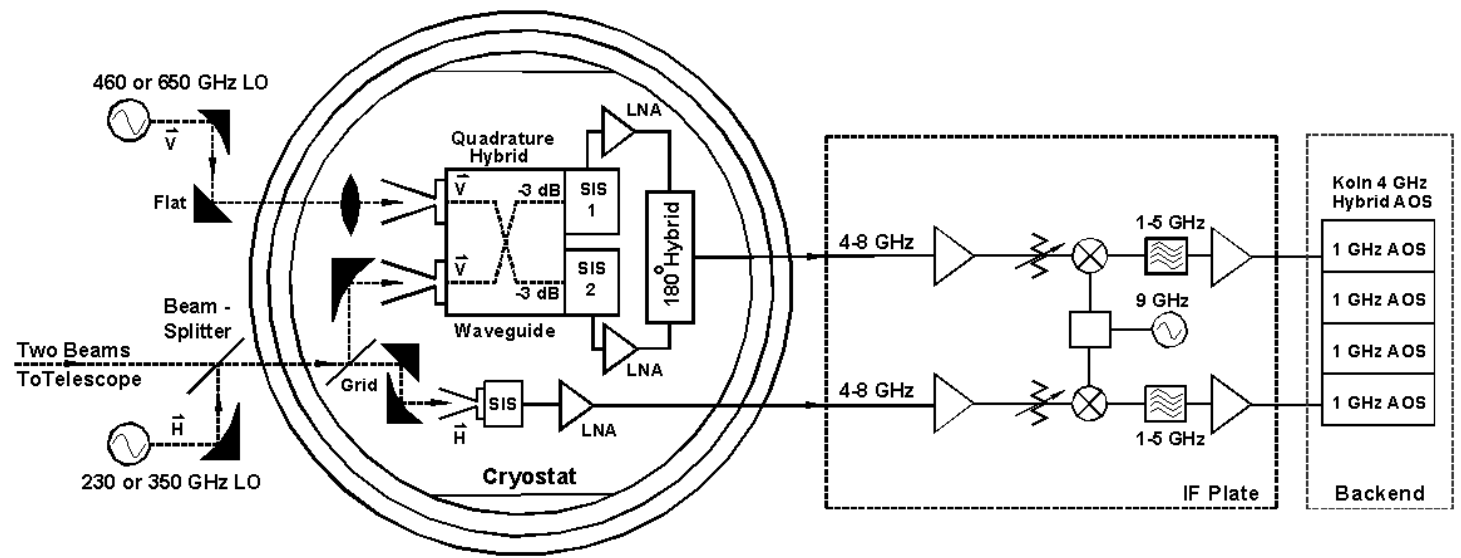

Figure 2. Block Diagram of the CSO Facility Instrumentation upgrade. Note that the high frequency channels (460and $660 \mathrm{GHz}$ ) are implemented in a balanced configuration. To minimize the noise and maintain high quality beams, only cooled reflecting optics is used in the signal path. 
Accompanying these new mixers is a bias control scheme that, once implemented, allows remote control of the instruments. An identical bias scheme is planned for the CASIMIR instrument on SOFIA. ${ }^{10}$ Aside from tunerless operation and wide IF bandwidth, the new instruments will be configured in such a way as to facilitate dual frequency mode observations. Referring to Fig. 2, a wire grid is used to separate out the random polarized input signal into its orthogonal components. The signal's horizontal and vertical component is then directed, via reflecting cooled $(4 \mathrm{~K})$ optics, to the appropriate mixers. As an additional feature, the high frequency channels $(380-520 \mathrm{GHz}$ and $580-720 \mathrm{GHz})$ will be implemented in a balanced arrangement. This has the advantage of using nearly all of the available LO power (currently only $\approx 10 \%$ is used), while at the same time rejecting $95-99 \%$ of the LO multiplier noise. In an actual unbalanced receiver $(\nu>500 \mathrm{GHz}$, beam splitter for LO injection), the LO noise adds an estimated $15-20 \mathrm{~K}$ to the overall receiver noise temperature. The low frequency receivers $(180-280 \mathrm{GHz}$ and $280-420 \mathrm{GHz})$ will remain unbalanced as LO power is readily available at these frequencies, and very thin film beam splitters can readily be used.

To minimize the noise and maintain high quality beams, only cooled reflecting optics are used in the signal path. An added advantage of this type of receiver configuration, is that it requires minimal change to the existing cryostat, telescope optics, and LO hardware. Because the Köln AOS consists of four $1 \mathrm{GHz}$ wide AOS channels, the IF bandwidth of each of the two receivers can be configured to use 25,50 , or $100 \%$ of the available $4 \mathrm{GHz}$ AOS band. This scheme has the advantage of potentially doubling the observing throughput as well as having co-aligned beams on the sky. The latter reduces calibration and pointing difficulties, especially for the high frequency receivers in mediocre weather.

\section{NEEDED TECHNOLOGY}

Significant progress on the upgrade of the correlation receiver and sidecab instrumentation hardware and software has been made. To achieve the required sensitivity and bandwidth, many of the needed components had to be developed "in house". A noted exception to this is the University of Chalmers InP based 2-3K cryogenic low noise amplifier. ${ }^{11,12}$ In the next section we briefly describe each of the following components:

- Tunerless Full-Height Waveguide Mixers with Large Fractional RF-Bandwidth

- Quadrature Waveguide Coupler with Large Fractional RF-Bandwidth

- Combined 4-8 GHz IF Matching Network, DC-Break, Bias Tee, and EMI Filter

- 4-8 GHz Low Noise Cryogenic Amplifier

- 4-8 GHz IF Gain Modules

- Allan Variance Study of SIS Receiver Instability

- Comprehensive Computer Control of the Bias Electronics

- New set of SIS Junctions, Section 4.10, Kovács et. al.

\subsection{Tunerless Full-Height Waveguide Mixers with Large Fractional RF-Bandwidth}

Many waveguide probe transitions have been proposed over the years, most of which have RF bandwidths of less than 35\%. To lower the input impedance, the majority of these designs require significant reductions in waveguide height. Unfortunately, reducing the height makes the machining of $\mathrm{THz}$ components difficult. It also increases RF loss, as the effects of poor surface quality are enhanced by the increased current density in the walls of the waveguide. Because nearly all mixer designs have some kind of integrated thin-film tuning structure, there is a need for an efficient waveguide to thin-film microstrip transition that covers at least one full waveguide band, and is also easily extendible to $\mathrm{THz}$ frequencies.

To date, the majority of SIS and HEB waveguide mixers have employed planar probes that extend all the way across the waveguide. ${ }^{14,16}$ An important reason for the popularity of this kind of design is the convenience with which the active device can be biased and the IF signal extracted. Unfortunately, this kind of "double-sided" (balanced) probe exhibits a rather poor RF bandwidth $(\leq 15 \%)$, when constructed in full-height waveguide. When the height of the waveguide is reduced by $50 \%$, the probe's fractional bandwidth improves dramatically to a maximum of about $33 \%{ }^{15}$ Reducing the height, however, can result in significant fabrication problems (e.g. cost) and increased RF loss, especially at frequencies near or above a terahertz. These results can be 

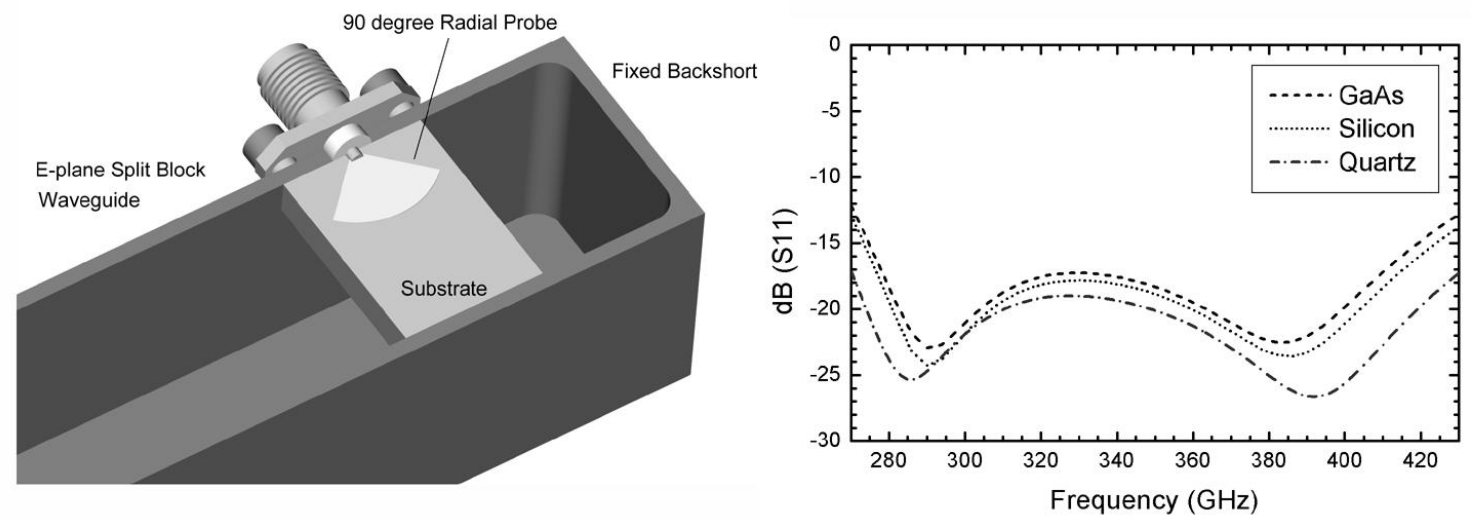

Figure 3. a) Probe configuration used in Withington's experiment. Orientation is parallel to the E-field of the TE10 waveguide mode, and in our case in the plane of the split-block. It is possible to rotate the probe by $90^{\circ}$ without much change in performance. b) Input return loss variation of a GaAs, Silicon, and Quartz based radial probe into a fixed load impedance (Table I).

Table 1. Radial Probe Parameters of Fig. 3

\begin{tabular}{|l|c|c|c|}
\hline \multicolumn{1}{|c|}{ Parameter } & Quartz & Silicon & GaAs \\
\hline Dielectric Constant $\left(\epsilon_{r}\right)$ & 3.78 & 11.9 & 12.9 \\
\hline Probe Radius $(\mu \mathrm{m})$ & 112 & 80 & 77 \\
\hline Backshort Distance $(\mu \mathrm{m})$ & 77 & 71 & 63 \\
\hline Substrate Width $(\mu \mathrm{m})$ & 180 & 120 & 120 \\
\hline Substrate Thickness $(\mu \mathrm{m})$ & 50 & 25 & 25 \\
\hline Probe Impedance $(\Omega)$ & $30+\mathrm{j} 0$ & $17-\mathrm{j} 6.0$ & $16-\mathrm{j} 5.5$ \\
\hline
\end{tabular}

understood in that the popular double-sided probe is essentially a planar variation on the well known Eisenhart and Khan waveguide probe. ${ }^{16}$ Borrowing from Withington's assessment, ${ }^{17}$ the real part of the probe's input impedance is influenced in a complex way by the parallel sum of individual non-propagating modal impedances, and as such, is frequency dependent. By reducing the height of the waveguide the effects of the non-propagating modes can be reduced, which has been done very successfully, for example, by Tong and Blundell et al.. ${ }^{15}$

An alternative approach is to use an asymmetric probe that does not extend all the way across the waveguide. For this kind of probe, referred to from now on as a "one-sided" probe, the modal impedances add in series. The real part of the input impedance only comes from the single propagating mode, and is relatively frequency independent. ${ }^{17}$

These probes are typically implemented in full-height waveguide, which minimizes conduction loss and eases fabrication complexity. Though a rectangular version of the "one-sided" probe is used quite extensively by microwave engineers ${ }^{18,19}$ and was introduced to the submillimeter community by Kerr et al. ${ }^{20}$ in 1990 , it is seen to be fundamentally different from the proposed radial-shaped probe. The radial probe described here represents an attempt to extend the use of radial modes, which are known to give superior broad band performance in thinfilm microstrip radial tuning stubs, as compared to rectangular stubs, to the microstrip to waveguide coupling problem. From a practical point of view, the radial probe can be made, quite naturally, to feed a thin-film microstrip line that has a small line width and thin insulator thickness. In the case of a rectangular probe, there would be a large geometrical discontinuity. Experimentally, we have found radial probes, implemented in the described thin-film configuration, give vastly superior performance over the more traditional approach.

In 1999, Withington et al. ${ }^{21}$ presented an extensive theoretical analysis of a "one-sided" rectangular probe in full-height waveguide. ${ }^{19,20}$ In this paper, alternative shapes of metallization were investigated, and very promising scale model measurements of a probe with a constant radius were presented. In these measurements 

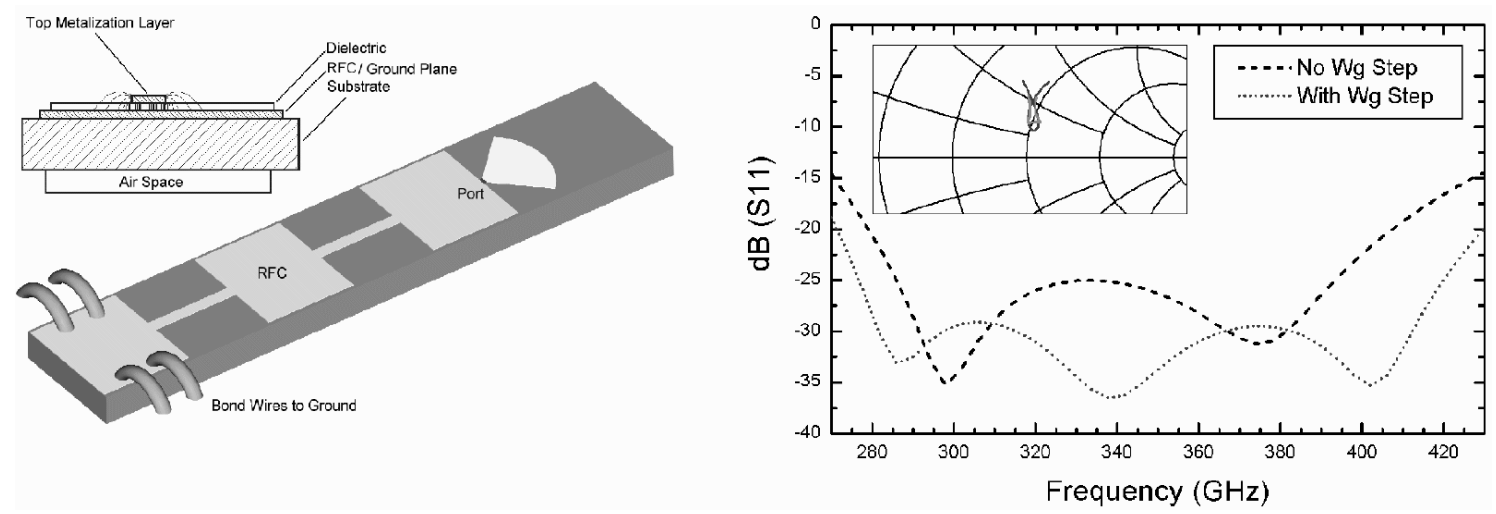

Figure 4. a) The RF choke provides a ground potential at the waveguide wall. The IF and bias lines run on top of the choke's metallization, which serves as a ground layer for thin-film microstrip or coplanar transmission lines, as shown on the inset (not to scale). The bond wires provide the DC bias return. b) Input return loss of the radial probe, with and without the capacitive waveguide tuning step. Here a $(31 \Omega)$ reference impedance is used, as this is typical of SIS tunnel junction mixers. The characteristic "tear drop" impedance locus of the probe collapses into a "star".

a $90^{\circ}$ radial fan was connected to a coaxial SMA connector that mounted to the side of split-block waveguide, as shown in Fig. 3. The probe was in the plane of the split block and oriented parallel to the electric field of the waveguide.

Extending a perfect ground all the way to the waveguide wall, as in the simple case of Fig. 3, is problematic in nearly all instances. Beam-lead techniques, are often used in GaAs Schottky diode multiplier processes, and are a viable option. ${ }^{22}$ This scheme, however, requires advanced and therefore expensive processing. Furthermore, the beam-lead process is not readily extendible to quartz substrates, which due to their low $\left(\epsilon_{r}=3.78\right)$ dielectric constant and RF loss are often the substrate of choice in the submillimeter wave region. An RF choke in the ground plane may be used to provide a good ground at the waveguide wall (Fig. 4). This idea can be extended to include membranes, ${ }^{23,24}$ which offer a good alternative to quartz substrates at frequencies near or above a terahertz.

If very broadband operation is required, a dramatic improvement in the probe's performance can be achieved by adding a simple capacitive waveguide tuning step, just in front of the probe. The added capacitive element collapses the characteristic probe impedance locus into a tiny "star", as shown in Fig. 4.
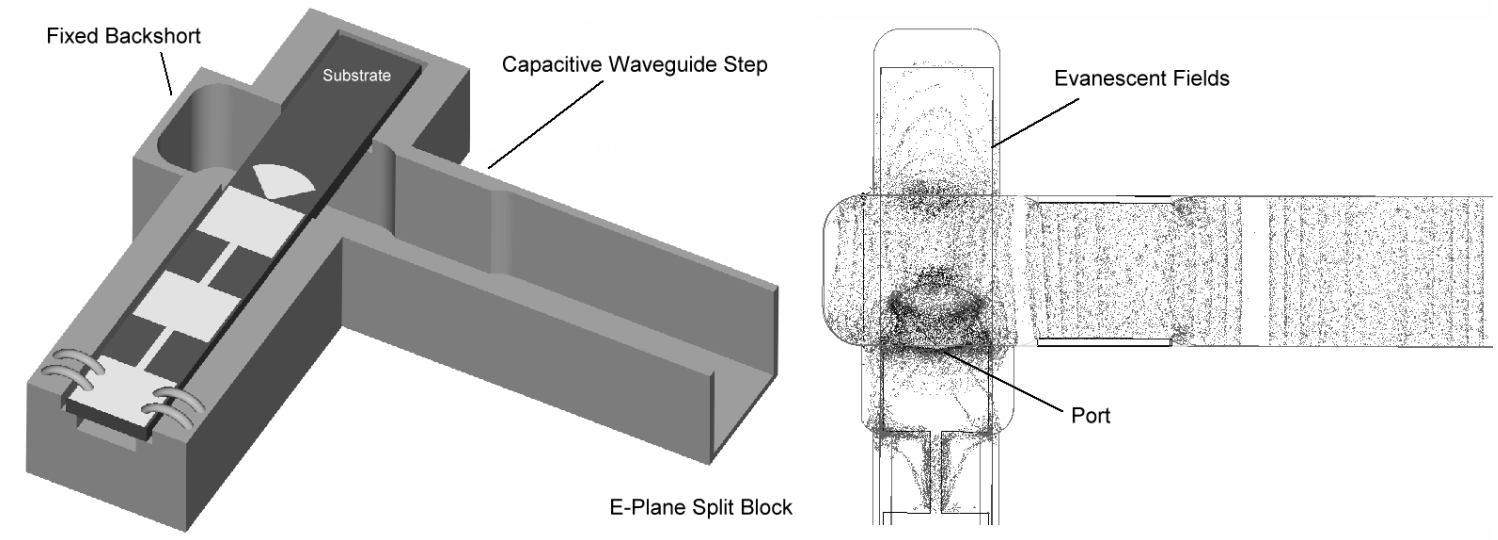

Figure 5. a) Inclusion of a capacitive tuning step in front of the radial probe. Though the physical size of the waveguide constriction is small $(\approx 15 \%)$, the reduction of the probe's input return loss and increase in bandwidth are dramatic. b) Electric field distribution in the waveguide and surrounding substrate material. 

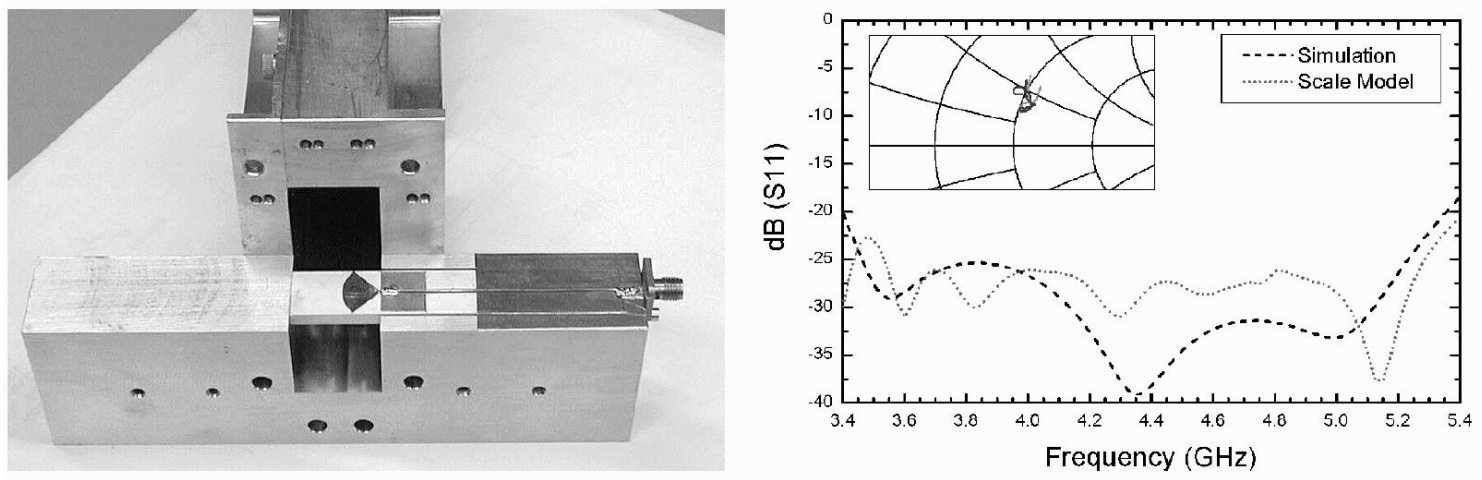

Figure 6. a) Scale model of the radial probe configuration, including RF choke to provide a ground reference at the waveguide wall. b) Measured and simulated return loss to a matched load. The input impedance of the modeled radial probe is $50+\mathrm{j} 20 \Omega$. These results include the two section RF choke and capacitive step in the waveguide.

Fig. 5 shows a diagram of a probe with the waveguide capacitive tuning step added. Typically, a $15 \%$ reduction in waveguide height is adequate to tune out most of the probe's residual impedance variation. The length of the step is on the order of the height dimension of the waveguide. Because some of the reactance in the probe is tuned out by the step, the distance between the substrate and backshort must be increased slightly: $\left(\Delta \approx 0.03-0.04 \% \lambda_{g}\right)$. This increase in distance is an additional advantage of using a waveguide step as it actually eases circuit and machining tolerances. Chamfered corners in the waveguide have no effect on the overall performance of the probe, as long as the position of the backshort is compensated for accordingly. The capacitive waveguide step does not affect the impedance locus of the probe.

\subsection{Scale model Verification}

To verify the simulations and obtained results, we ran a series of S-band scale model measurements. The experimental arrangement is shown in Fig. 6. For calibration standards we used 1, 50, and 100 Ohm chip resistors. After making a small adjustment for the physical length of the chip resistors, we established the phase reference at the edge of the waveguide. The substrate material was Stycast with a measured dielectric constant of $\epsilon_{r}=4.05$ and a loss tangent $\delta=0.020$. The presented results verify the accuracy of the HFSS 3-D Electromagnetic field simulations. ${ }^{26}$ The reactive component of the probe's impedance can be reduced by thinning the substrate and/or increasing the airgap under the first section of the RF choke.

\subsection{Example of a fixed-tuned $270-430 \mathrm{GHz}$ design}

As a summary of our work, we present, in Fig. 7, a comprehensively modeled design for a 270-430 GHz SIS waveguide mixer. The mixer block consists of a full-height, fixed tuned waveguide, which excites a supercon-

Table 2. Parameters used for the 270-430 GHz Design

\begin{tabular}{|l|c|}
\hline \multicolumn{1}{|c|}{ Parameter } & \\
\hline Substrate material & Quartz \\
\hline Waveguide size $(\mu \mathrm{m})$ & $600 \times 280$ \\
\hline Probe radius $(\mu \mathrm{m})$ & 118 \\
\hline Substrate width $(\mu \mathrm{m})$ & 200 \\
\hline Substrate thickness $(\mu \mathrm{m})$ & 50 \\
\hline Air Height above substrate $(\mu \mathrm{m})$ & 25 \\
\hline Air Height below substrate $(\mu \mathrm{m})$ & 50 \\
\hline Backshort distance $(\mu \mathrm{m})$ & 103 \\
\hline Probe impedance $(\Omega)$ & $47+\mathrm{j} 3$ \\
\hline
\end{tabular}




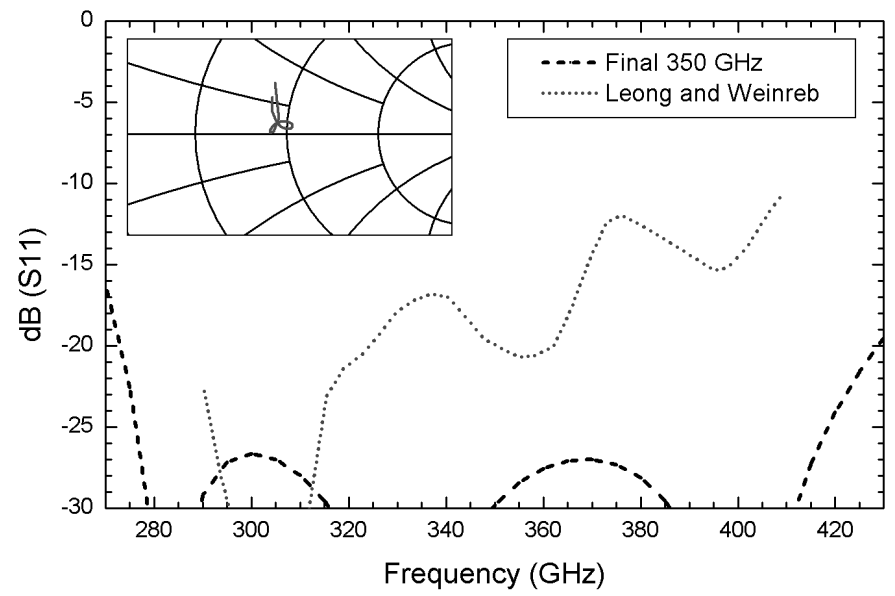

Figure 7. The predicted input return loss of a fixed tuned full-height SIS mixer block. Also shown is the predicted performance from a rectangular probe as reported by Leong and Weinreb et al. [17]. The fractional bandwidth of the $350 \mathrm{GHz}$ full height waveguide radial probe is $\approx 45 \%$. Refer to Table 2 and the text for details.

ducting tunnel junction (SIS) heterodyne detector. The mixer block employs a capacitive tuning step $82 \mu \mathrm{m}$ in front of the probe. A 12-13\% reduction in the height of the waveguide with a length of $240 \mu \mathrm{m}$ was found to be optimal. The substrate width $(200 \mu \mathrm{m})$ was dictated by the need to accommodate the $118 \mu \mathrm{m}$ radial probe. This in turn required the use of an air-gap underneath the substrate, to raise the cutoff frequency of the dielectric-loaded IF channel to above the RF operating frequency of the receiver.

\subsection{Quadrature Waveguide Coupler with Large Fractional RF-Bandwidth}

For the correlation receiver to work, it requires either a $180^{\circ}$ or $90^{\circ}$ phase shift between the input ports. Considering ease of fabrication we decided to use a $90^{\circ}$ branch line waveguide coupler. The design is based on a narrower bandwidth split block version, developed for the ALMA project by Claude and Cunningham et al.. ${ }^{25}$ Design and optimization of the wideband quadrature hybrid coupler were done in HFSS. ${ }^{26}$ From $280-420 \mathrm{GHz}$, the coupled power imbalance is $0 \pm 1 \mathrm{~dB}$, while the predicted phase imbalance is less than $90 \pm 2^{\circ}$. Design parameters are compiled in Table 3, and the predicted performance is shown in Fig. 10.

In order to achieve the required bandwidth, phase, and coupling it was necessary to maximize the number of branch lines (n). It was observed that:

$$
n * S=\frac{\lambda_{g}}{2}
$$

Where $S$ is the width of one of the branch lines, and $n$ the number of sections. Keeping the hybrid manufacturable we decided, after consultation with Custom Microwave Inc. ${ }^{27}$ on the use of 8 sections. To maximize the

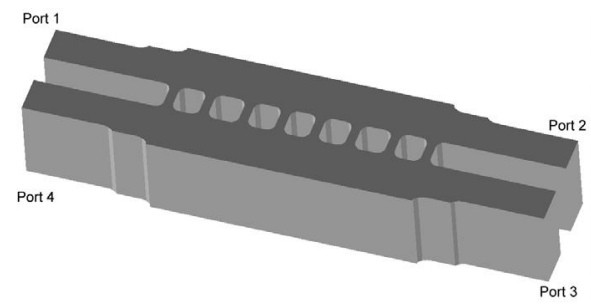

Figure 8. Branch line waveguide coupler ( 8 sections). Port1 is the input port, Port 2 and 3 the output (coupled) ports, and port 4 the isolation port. Refer to Table 3 for details. 
thickness of the branch lines, the waveguide width (b-dimension) of the coupler has been increased by a $32.5 \%$. Increasing the waveguide width beyond this has the effect of exciting the next higher mode, thereby degrading the high frequency performance of the coupler. The separation of the two waveguides is $\approx \lambda_{o} / 4$ as measured at the center of the band.

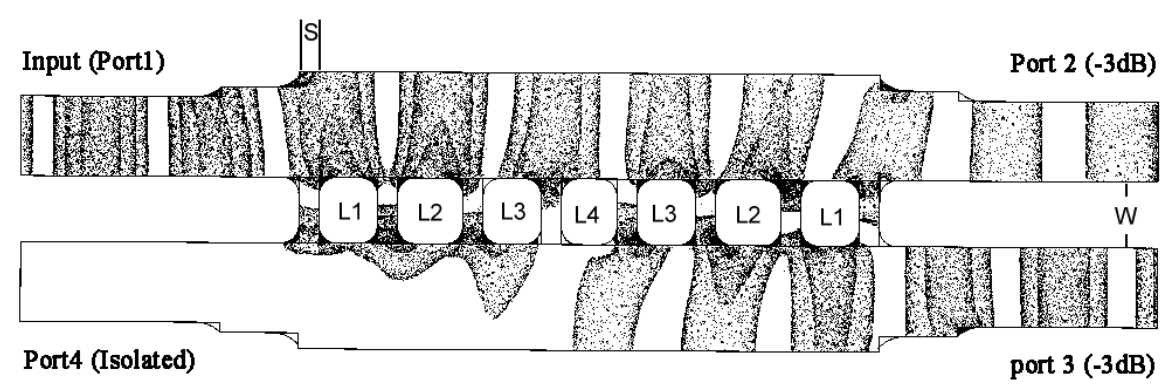

Figure 9. Electric field distribution in the quadrature waveguide coupler. The phase difference between port 2 and 3 is $90 \pm 1.5^{\circ}$.

Table 3. Hybrid Coupler Parameters used in Fig. 10

\begin{tabular}{|c|c|c|c|c|c|c|c|}
\hline $\begin{array}{c}\text { a-dim } \\
(\mu \mathrm{m})\end{array}$ & $\begin{array}{c}\text { b-dim } \\
(\mu \mathrm{m})\end{array}$ & $\begin{array}{c}\mathbf{S} \\
(\mu \mathrm{m})\end{array}$ & $\begin{array}{c}\text { L1 } \\
(\mu \mathrm{m})\end{array}$ & $\begin{array}{c}\text { L2 } \\
(\mu \mathrm{m})\end{array}$ & $\begin{array}{c}\mathbf{L 3} \\
(\mu \mathrm{m})\end{array}$ & $\begin{array}{c}\mathbf{L 4} \\
(\mu \mathrm{m})\end{array}$ & $\begin{array}{c}\text { W } \\
(\mu \mathrm{m})\end{array}$ \\
\hline 680 & 280 & 71 & 205 & 230 & 205 & 195 & 230 \\
\hline
\end{tabular}

$S$ denotes the branch line width, $L$ the length between the branch lines, and $W$ the separation between the two waveguides.
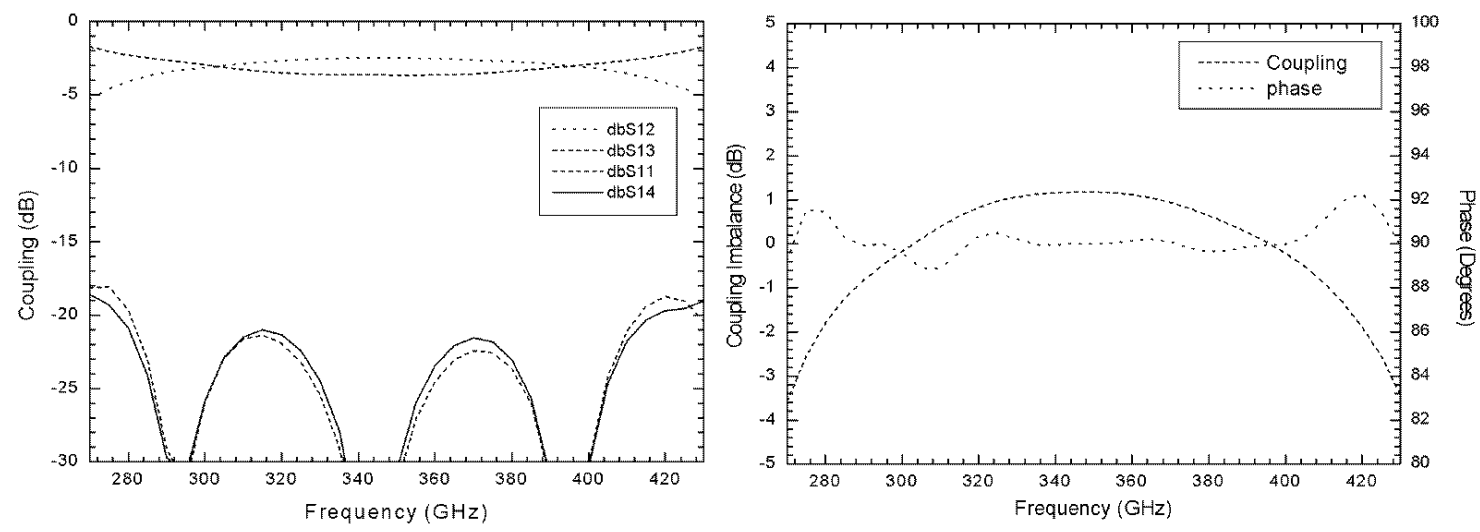

Figure 10. a) Predicted coupling performance. b) Delta phase and power imbalance of the quadrature hybrid coupler.

\subsection{Combined 4-8 GHz IF Matching Network, DC-Break, Bias Tee, and EMI Filter}

In a practical receiver configuration, the mixer is terminated into a desired IF load impedance, the bias lines EMI filtered and injected via a bias Tee, and the IF output DC-blocked. The latter is usually accomplished with a small capacitor, by means of either a soldered contact or with wire bonds. Unfortunately series resonance of real physical capacitors are, by design, often located very near the edge of the IF band. Moreover, since the dc-blocking capacitor passes the mixer IF output, failure will be catastrophic. Indeed, component failure can come in may ways. The most obvious one is perhaps stress due to repeated thermal cycling. 
Table 4. Coupling Parameters of Fig. 12

\begin{tabular}{|c|c|c|c|c|}
\hline Name & $\begin{array}{c}\mathbf{W} \\
(\mu \mathrm{m})\end{array}$ & $\begin{array}{c}\mathbf{L} \\
(\mu \mathrm{m})\end{array}$ & $\begin{array}{c}\mathbf{S} \\
(\mu \mathrm{m})\end{array}$ & $\begin{array}{c}\text { HC } \\
(\mu \mathrm{m})\end{array}$ \\
\hline dbS21block1 & 450 & 5.50 & 100 & 635 \\
\hline dbS21block7 & 480 & 5.72 & 125 & 525 \\
\hline
\end{tabular}

$W$ denotes the width of the coupled lines, $L$ their length, $S$ the spacing, and $H C$ the cavity depth.

For a viable alternative, we looked into the use of parallel coupled suspended microstrip lines. ${ }^{28-30}$ As shown in Fig. 11, the suspended coupled microstrip lines act as a compact bandpass filter. For this filter to work, the ground plane directly underneath the filter has been removed, and the IF board positioned on top of a machined cutout (resonant cavity). There are several discontinuities in this structure. When combined, they form the bandpass filter poles.

The advantages are, simplicity of design (only one lithography step), and significantly improved reliability. The disadvantage in some cases may be its size, $\lambda_{g} / 4$.

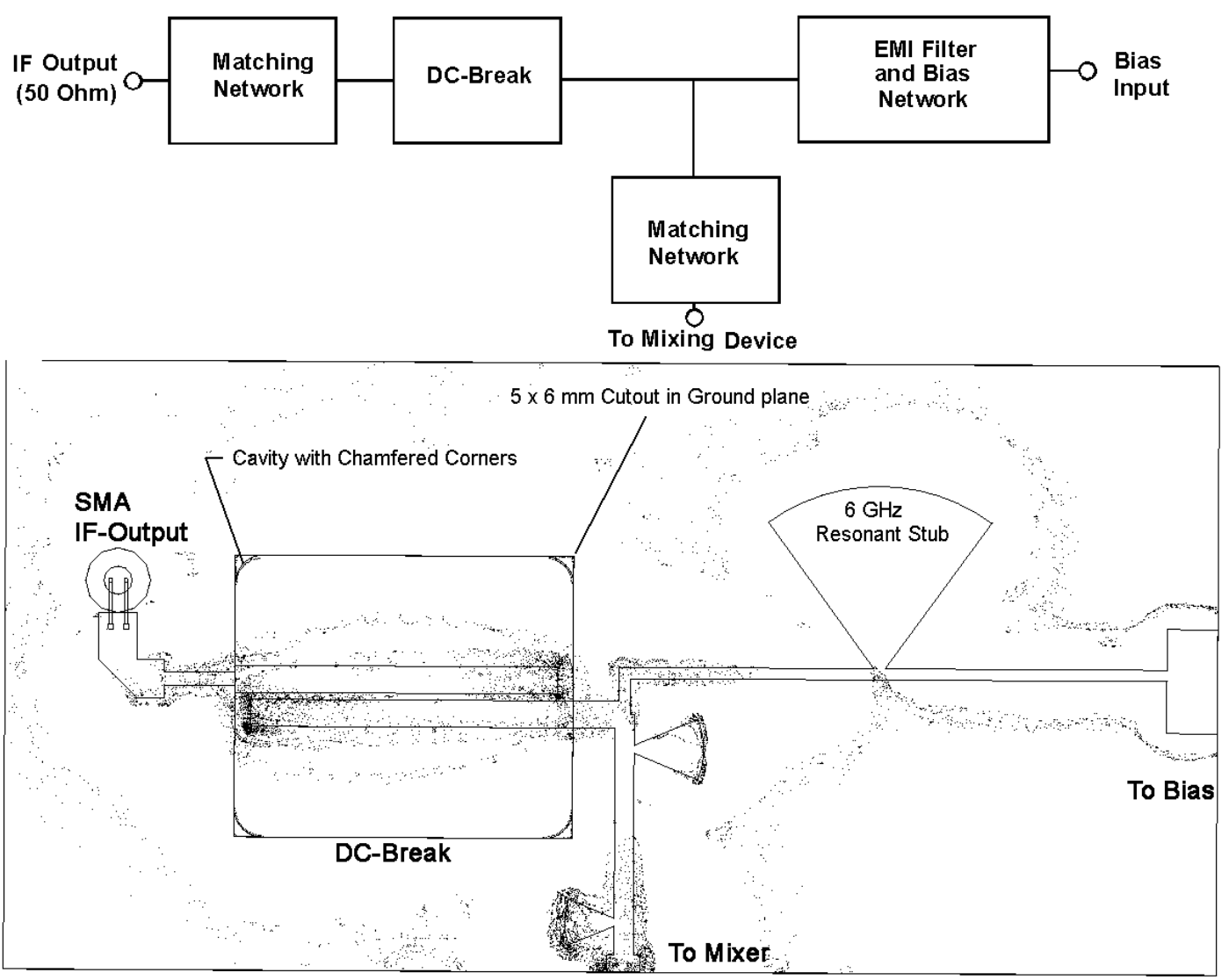

Figure 11. Example of a combined IF match, DC-break, Bias Tee, and EMI filter on the bias line. All these components are needed to properly bias the mixer, and provide the desired IF load impedance.

Fig. 12 presents two design examples of the DC-break. In the case of "dbS21block1", we traded the input return loss for bandwidth. While in the case of "dbS21block7", the input return loss (dBS11) was optimized $\left(\mathrm{HFSS}^{26}\right)$. Details of the blocking filter summarized in Table 4 . The spacing $S$, and cavity depth $H C$ set the coupling. Their tolerance values should be held to $\pm 5 \%$. 

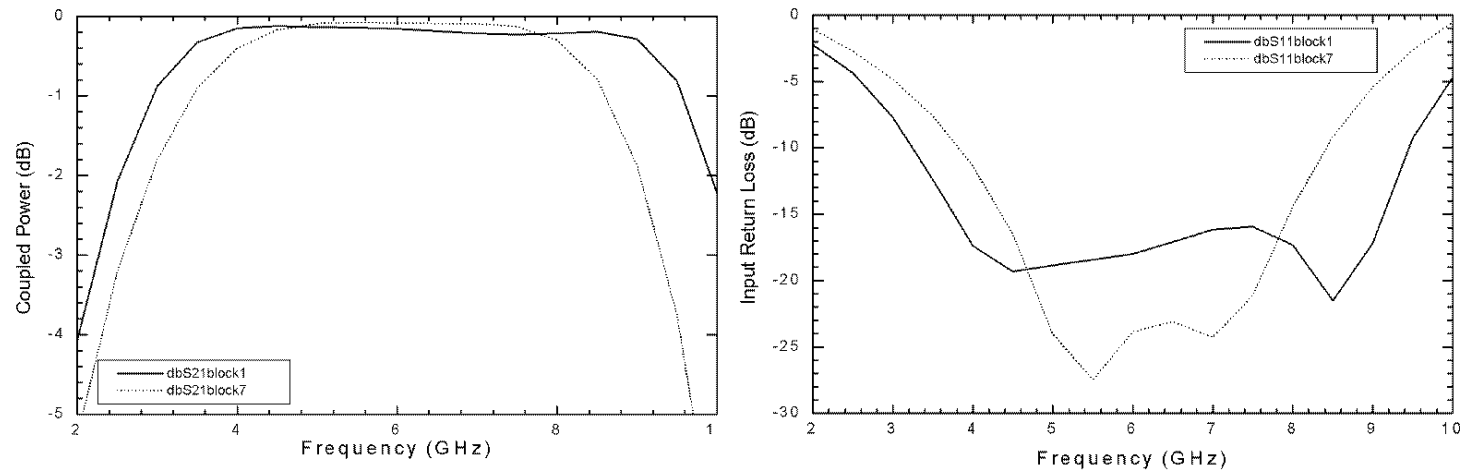

Figure 12. HFSS simulated DC-Break performance. dbS21block1 was optimized for bandwidth, dbS21block7 for input return loss. The conductor is assumed to have the conductivity of room temperature gold. The substrate is Alumina, with an $\epsilon_{r}=9.80$, a loss tangent of 0.0018 , and thickness of $635 \mu \mathrm{m}$. Refer to Table 4 for details.

\subsection{4-8 GHz Low Noise Cryogenic Amplifier}

In collaboration with Chalmers University in Sweden and NASA's JET Propulsion laboratory, we have acquired extremely low noise (2-3K) 4-8 GHz Indium Phosphide (InP) cryogenic low noise HEMT amplifiers. ${ }^{11,12}$ The DC Power consumption and input return loss of these amplifiers are excellent, $\approx 10 \mathrm{~mW}$ and $-18 \mathrm{~dB}$ respectively. This means that the amplifier can be mounted on the LHe stage, and that cryogenic isolators may not be needed (though they are available commercially from Pamtek ${ }^{13}$ ). The amplifier gain is $\approx 25 \mathrm{~dB}$ (2 stages), and we are considering the use of low input return loss MMIC's for some additional cryogenic amplification.

\subsection{4-8 GHz IF Gain Modules}

Room temperature amplification is accomplished by means of custom designed, CTT Inc. ${ }^{32}$ fabricated and tested, gain modules. The overall gain of these modules is variable up to $60 \mathrm{~dB}$, the in band gain flatness $\pm 1.5 \mathrm{~dB}$, the input return loss $\leq 15 \mathrm{~dB}$, and the noise figure $2.8 \mathrm{~dB}$.

\subsection{Allan Variance Study of SIS Receiver Instability}

To achieve the required $85 \mu \mathrm{K}$ of noise (100 MHz resolution BW), it is absolutely essential to understand (and minimize) causes of receiver instability. In 2000 we undertook such a study, ${ }^{31}$ and many of the findings have been incorporated in the presented designs.

\subsection{Comprehensive Computer Control of the Bias Electronics}

Control of the new receivers will be much simpler than with the existing tuned waveguide receivers. Nevertheless there are a lot of parameters that will need to be set (or optimized). To automate these tasks, we are developing the hardware and software that will allow near total automation of the tuning process. An added advantage is that the instruments can then be accessed remotely, if problems were to arise. The actual electronics consists of a set of analog cards, each backed with a digital card. The purpose of the analog cards are to bias the SIS junctions, the LNA's, set the magnetic field to suppress the Josephson effect, control the CTT Inc. ${ }^{32}$ IF gain modules, and to measure the IF output power level of the mixers

The analog bias electronics design has been finished, and the cards are in the process of being fabricated. First generation digital cards have also been developed, and are currently used to communicate with the ADCs and digital potentiometers on analog cards. The software has many components. The first of which resides in a FPGA on the digital card. It is programmed to receive commands, retrieve data from the ADCs, and set the digital potentiometers that reside on the analog cards. The second software package resides in the electronics chassis, and parses commands received over the Ethernet from a database server computer. The third software package resides on a database server, and provides a GUI receiver control interface for the user. 


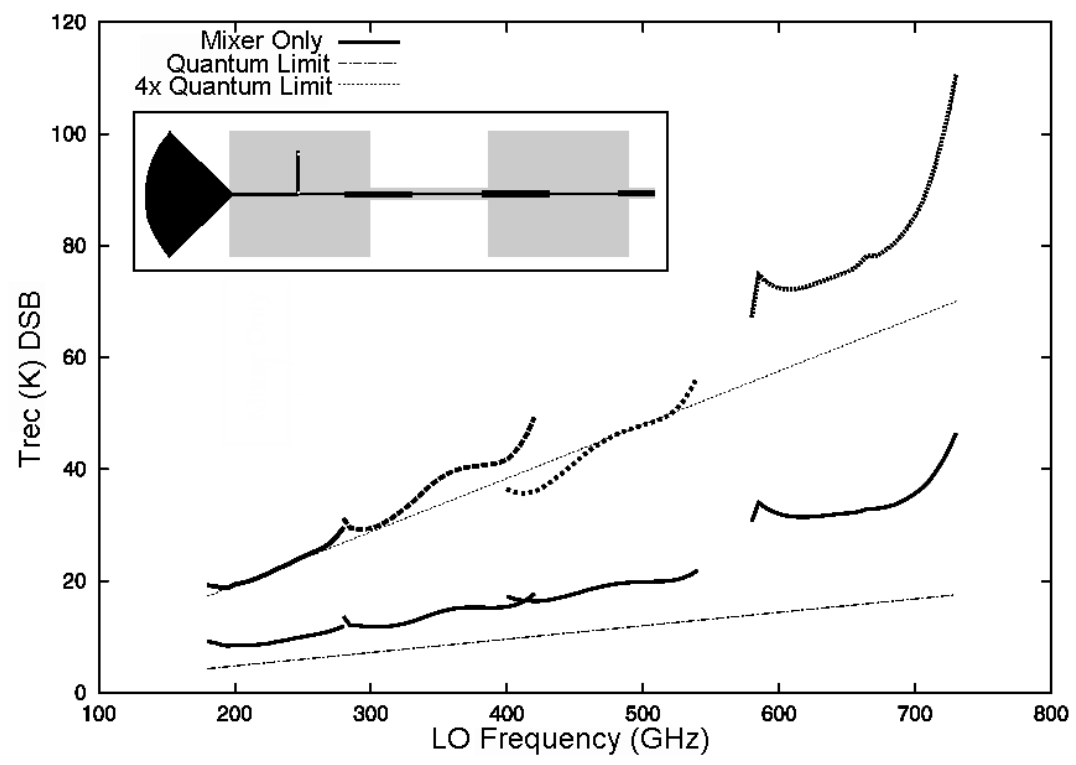

Figure 13. Simulated Rx noise temperature for the twin junction designs. Inset: Detail of the new niobium based SIS junctions. Design is for a 1-12 GHz IF output, though just 4-8 GHz will be used for the current upgrade. The higher frequency receivers are implemented in a balanced configuration.

\subsection{New set of SIS Junctions}

Designs of the niobium based SIS junctions (4 bands) is essentially finalized. The new devices will all share the same $50 \mu \mathrm{m}$ Quartz wafer. The designs use twin SIS junctions with $14 \mathrm{kA} / \mathrm{cm}^{2}$ current density, and AlN barriers. Supermix ${ }^{6}$ a flexible software library for high-frequency circuit simulation has been used exclusively in the design process.
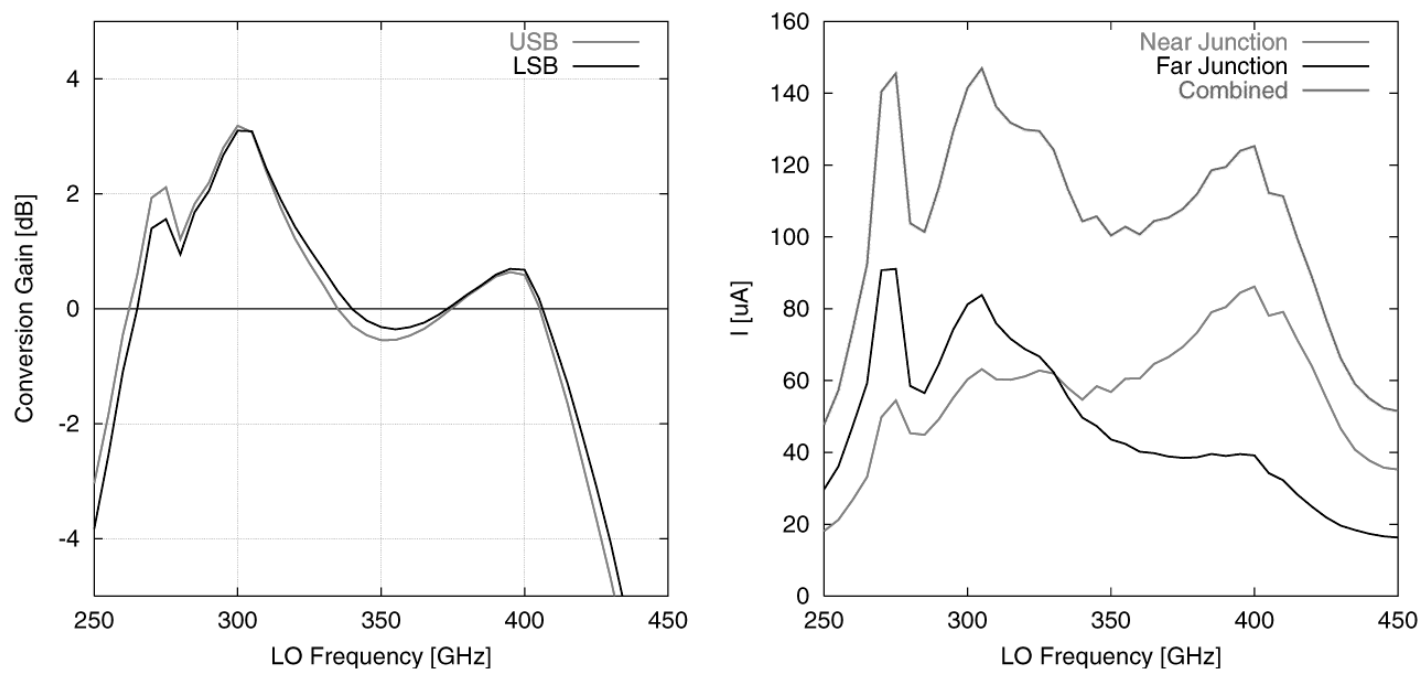

Figure 14. a) Simulated mixer conversion gain of the twin junction design. Over the 280-420 GHz frequency band the mixer gain is expected to be $\geq 1$. b) Simulated direct detection (FTS) response of the individual and combined SIS junctions (refer to Fig. 13 for the junction layout). Note the strong correlation between the heterodyne and direct detection "measurements". 
Both RF and IF matching is realized on chip, yielding a flat IF response out to high (>12 GHz) IF frequencies. All designs have been optimized for maximal conversion gain and minimal noise temperatures across the entire operational band. In Fig. 13, we show the simulated receiver and mixer noise from 180-720 $\mathrm{GHz}$ in 4 waveguide bands. To obtain a realistic estimate of the receiver noise, we used the measured optics losses of the present receivers. In the case of the balanced mixers, receiver noise is lowered 5-10K due to the elimination of the Local Oscillator noise. The designs have also been checked for IF saturation. Supermix can further be used to determine optimal bias and pumping conditions, while at the same time avoiding instability in the SIS mixer. Instability can result in increased RF and IF reflection, which in turn is likely to cause poor baseline subtraction (data). These optimal values can be set by the computer controlled bias electronics as discussed in Section 4.9. The predicted mixer conversion gain and direct detection response of the twin junction design is shown in Fig. 14. There is a strong correlation between the expected heterodyne conversion gain and direct detection response, a phenomenon widely observed in actual SIS mixer measurements.

\section{CONCLUSION}

Upgrade plans to the Caltech Submillimeter Observatory (CSO) have been presented. In the near future, the existing waveguide receivers will be upgraded with very broad bandwidth, tunerless SIS mixers. The IF for the new system is 4-8 GHz. Plans have been made to use mixers in a balanced configuration for higher frequency (460 and $650 \mathrm{GHz}$ ) receivers. This has the advantage that all the LO power is used, greatly simplifying the LO tuning process. An added benefit of this scheme is that LO noise (15-20K) is cancelled in the receiver.

Finally, good progress has been made on the development of a 280-420 GHz continuous comparison receiver, for the detection of very faint (high-z) extragalactic sources. Much of the required hardware, needed for the new instrumentation has been developed at Caltech, and is now in a state where it can be fabricated. Barring unforeseen problems, installation should commence toward the summer of 2003. The actual upgrade may take several years, as all IF hardware at the telescope will need to be replaced to accommodate the new 4-8 GHz IF.

\section{ACKNOWLEDGEMENTS}

We wish to thank Sander Weinreb of JPL for very helpful discussions on "one-sided" waveguide probes. This work was supported in part by NSF Grant\# AST-9980846.

\section{REFERENCES}

1. R.Schieder, O.Siebertz, F.Schloeder, C.Gal, J.Stutzki, P.Hartogh, V.Natale, "Wide-Band Spectrometer for HIFI-FIRST", Proc. of "UV, Optical, and IR Space Telescopes and Instruments", J.B. Breckinridge, P.Jakobsen Eds., SPIE 4013, 313-324, 2000.

2. R.Schieder, J.Horn, O.Siebertz, M.Klumb, J.Frerick, V.Tolls "Acousto-Optical Spectrometers in Space", The 30th ESLAB Symposium on Submillimetre and Far-Infrared Space Instrumentation, ESTEC, Noordwijk, The Netherlands, Sept. 24-26, 1996, ESA SP-388, 187.

3. J.Horn, O.Siebertz, F.Schmilling, C.Kunz, R.Schieder, G.Winnewisser; "A 4X1 GHz Array Acousto-Optical Spectrometer", Experimental Astronomy Vol. 9, 17-38, 1999)

4. A. I. Harris, and J. Zmuidzinas, "A Wideband Lag Correlator for Heterodyne Spectroscopy of Broad Astronomical and Atmospheric Spectral Lines" "Rev. Sci. Instrumentation, Vol 72, No. 2, pp. 1531-1538, 2001

5. A. I. Harris, Proceedings of SPIE, Millimeter and Submillimeter Detectors,Kona, HI. 2002.

6. J. Ward, F. Rice and J. Zmuidzinas "Supermix: a flexible software library for high-frequency circuit simulation, including SIS mixers and superconducting components", Tenth International Symposium on Space Terahertz Technology, University of Virginia, Charlottesville, VA, March 1999.

7. C.R. Predmore, N. R. Erickson, G.R. Huguenin, and P. Goldsmith, "A Continuous Comparison Radiometer at 97 GHz" IEEE, Microwave Theory and Techniques, Vol MTT-33, No. 1, January 1985.

8. J.Lamb "SSB vs. DSB for Submillimeter Receivers", Alma Memo 301.

9. J.D. Kraus, "Radio Astronomy", 2nd Edition, pp $7-8$ 
10. M. L. Edgar and J. Zmuidzinas, "CASIMIR, a Submillimeter Heterodyne Spectrometer for SOFIA" Proceedings of SPIE, Airborne Telescope Systems, Vol. 4014, pp. 31-42, Munich, Germany 2000

11. N. Wadefalk, A. Mellberg, I. Angelov, E. Choumas, E. Kollberg, N. Rorsman, P. Starski, J. Stenarson, and H. Zirath, "Cryogenic, Wideband, Ultra-low noise IF-Amplifiers operating at Ultra-Low DC-Power," Submitted to IEEE MTT for Publication (2002).

12. Private Communication.

13. Pamtek 4053 CalleTesoro, Camarillo, Ca 93012, USA.

14. J. W. Kooi , M. Chan, B. Bumble, and T. G. Phillips, "A low noise $345 \mathrm{GHz}$ waveguide receiver employing a tuned $0.50 \mu \mathrm{m}^{2} \mathrm{Nb} / \mathrm{AlO}_{x} / \mathrm{Nb}$ tunnel junction," Int. J. IR and MM Waves, vol. 15, No. 5, May 1994.

15. Tong C-Y. E., Blundell R, Paine S, "Design and characterization of a 250-350-GHz fixed-tuned superconductor-insulator-superconductor receiver", IEEE, Microwave Theory and Techniques, Vol MTT44, pp. 1548-1556, Sept. 1996.

16. R. L. Eisenhart and P. J. Khan, "Theoretical and experimental analyses of a waveguide mounting structure", IEEE, Microwave Theory and Techniques, Vol MTT-19, pp. 706-717 (1971).

17. S. Withington, and G. Yassin, "Analytical expression for the input impedance of a microstrip probe in waveguide," newblockInt. J. IR and MM Waves, Vol. 17, pp. 1685-1705, Nov. 1996.

18. Y-C Leong, and S. Weinreb "Full-band Waveguide-to-microstrip probe transitions" IEEE, Microwave Theory and Techniques, Digest of Papers, Anaheim, CA, June 13-19, 1999.

19. J.H.C. van Heuven "A new integrated waveguide-microstrip transition", IEEE, Microwave Theory and Techniques, Vol MTT-24, pp. 144-147, March 1976.

20. A. R. Kerr and S. K Pan, "Some recent developments in the design of SIS mixers,", Int. J. IR and MM Waves, Vol. 11, No. 10, pp. 1169-1187, Nov. 1990.

21. S. Withington, G. Yassin, J. Leech, and K. G. Isaak, "An accurate expression for the input impedance of one-sided microstrip probes in waveguide", Tenth International Symposium on Space Terahertz Technology, Charlottesville, March 1999.

22. E. Schelecht, G. Chattopadhyay, A. Maestrini, A. Fung, S. Martin, D. Pukala, J. Bruston, and I. Mehdi, "200, 400, and $800 \mathrm{GHz}$ Schottky diode substrateless multipliers: Design and Results" 2001 IEEE, MTT-S International Microwave Symp. Digest, Phoenix, Az, pp1649-1652, May 2001.

23. J.W. Kooi, J. Pety, B. Bumble, C.K. Walker, H.G. LeDuc, P.L. Schaffer, and T.G. Phillips, "A 850 GHz Waveguide Receiver employing a Niobium SIS Junction Fabricated on a $1 \mu \mathrm{m} \mathrm{Si}_{3} \mathrm{~N}_{4}$ Membrane," IEEE Transactions on Microwave Theory and Techniques, Vol. 46, No. 2, pp151-161, February 1998.

24. J. W. Kooi, C.K. Walker, J. Hesler, "A broadband suspended membrane waveguide to microstrip transition for THz Applications," 9th International Conference on Therahertz Electronics, University of Virginia, Oct. $15-16,2001$.

25. S.M.X Claude and C.T. Cunningham, "Design of a Sideband-Seperating Balanced SIS Mixer Based on Waveguide Hybrids," Alma Memo 316, September 2000

26. Ansoft Corporation Four Station Square, Suite 200, Pittsburgh, PA 15219-1119, USA

27. Custom Microwave Incorporated Custom Microwave, Inc., 940 Boston Avenue Longmont, CO 80501 USA

28. W. Menzel, L. Zhu, K. Wu, F. Bögelsack Compact Broadband Planar Couplers, $31^{\text {st }}$ European Microwave Conference, London, UK, Sept. 25-27, 2002.

29. G. de Lange, SRON, NL, Private Communication

30. H. Golstein, SRON, NL, Private Communication

31. J.W. Kooi, G. Chattopadhyay, M. Thielman, T.G. Phillips, and R. Schieder, "Noise Stability of SIS Receivers," Int J. IR and MM Waves, Vol. 21, No. 5, May, 2000

32. CTT Incorporated 3005 Democracy Way, Santa Clara, CA 95054, USA 